\title{
Butler: A Visitor Guide Robot
}

\author{
Sung-on Lee, Nak-ju Do†, Dong To Nguyen, \\ Myung Hwangbo, Bum-jae You, and Sang-rok Oh \\ Intelligent Robot Center, Korea Institute of Science and Technology (KIST) \\ $†$ Mech. Eng. Dep., Pohang University of Science and Technology (POSTECH) \\ solee@kist.re.kr
}

\begin{abstract}
This paper describes the major components of software architecture of an autonomous visitor guide robot with the brief explanation of its hardware system. This robot, "Butler," was deployed in a real office environment, Intelligent Robot Research Center at KIST, where it guided many visitors successfully. To make the robot navigate the office and interact with visitors without problems, many challenges such as navigation in dynamic environments and interaction through various methods should be overcome. In this paper, the solutions for those problems are illustrated and our conclusion about this project is discussed.
\end{abstract}

Keywords: guide robots, mobile robots, service robots, navigation, HRI

\section{INTRODUCTION}

This paper describes Butler, a visitor guide robot, about the major components of software architecture of an autonomous visitor guide robot with the brief explanation of its hardware system. This robot was deployed in a real office environment, Intelligent Robot Research Center at KIST, where it guided many visitors successfully. To make the mobile robot operate safely and robustly in the office, many functions such as localization, collision avoidance, and path-planning are essential as well as a proper coordination of those functions. A user friendly interface is another very important part when developing a guide robot which needs to interact with people continually. Butler supports various easy-touse interfaces like voice, touch screen, PC vision.

The robot's major components can be thought as three parts like the following:

Hardware: Butler's mobile platform adopted omnidirectional wheel mechanism; all wheels are castors. Modularization based on the network-based system was adopted when implementing the controller systems. The shape and the height of the robot were also determined by considering user's convenience.

Navigation: Butler's navigation modules can be divided into three main parts: localization, obstacle avoidance, and path-planning. Not only every part should do well its own function, but also all parts should be controlled to cooperate with one another properly by well-organized coordination architecture.

Human-Robot Interface (HRI): We introduced and implemented a multi-modal HRI system, which supported various HRI methods such as voice, vision, touch screen GUI. The system was designed so that all types of human-robot interaction can work simultaneously in both directions.

In the following sections, we will discuss each part in more detail.

\section{HARDWARE SYSTEMS}

Butler's omni-directional wheel mechanism enables the robot to move to every direction with the redundant freedom of its orientation at any time. This function makes it possible for the robot to guide the visitor to a given position with simultaneously looking him/her in the face for continuous interaction. For the omni-directional mechanism, the robot employed three castor wheel type since omnidirectional mobile robot with three castor wheels is more efficient than omni-directional mobile robot with Swedish wheels. Two motors for each castor were assigned and total six motors were used to avoid any singularity which happens in this type robot when less than four motors are used and to give redundant actuation which provides mobile system 
(a)

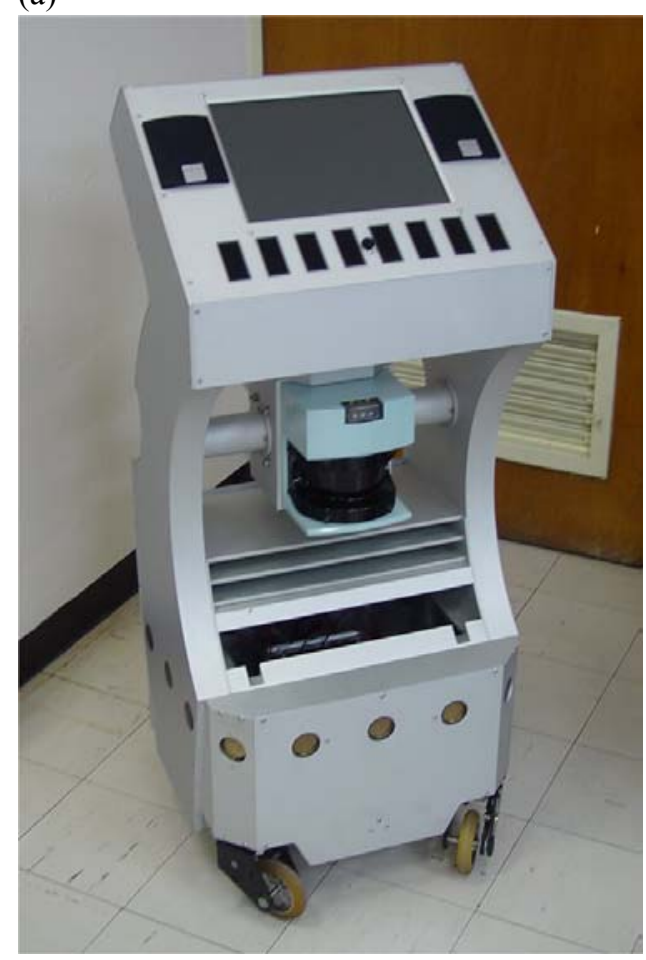

(b)

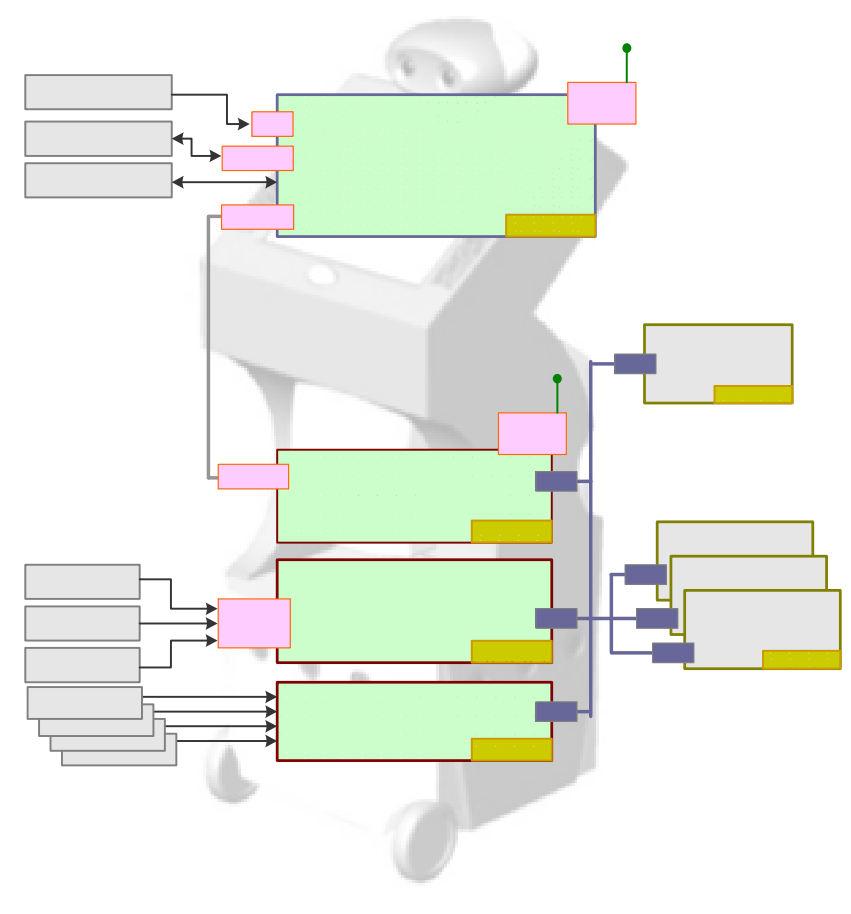

Figure 1. (a) Butler. (b) The architecture of controller.

with many beneficial characteristics such as good load distribution and fault-tolerant capability[5].

When it comes to Butler's electrical equipments, it consists of 2 major parts; the human interface part and the robot control part. Human interface was implemented with a notebook equipped with a touch screen, a microphone, a USB camera and speakers in the kiosk-like mobile robot. The robot control part was developed with a networked-based distribution systems using CAN(Controller Area Network) with maximum speed of $1 \mathrm{MHz}$. Advantages of the network-based architecture are parallel developing process, isolation of a problem, easy modification or repair. The networked based distribution systems are composed of hardware-independent modules such as a navigation-localization module, a central control module and a sensor module. Functions for each module and the overall controller system configuration are shown in figure 1(b).

\section{NAVIGATION}

\subsection{Kinematics for control}

From the relation between the mobile robot's velocity $(\dot{x}, \dot{y}, \dot{\theta})$ and each castor's joint angle, joint angle velocity and wheel velocity $(\beta, \dot{\beta}, \dot{\varphi})$ in figure 2 , the following equations can be obtained for all the castors[x].

$$
d \dot{\beta}=-\cos (\alpha+\beta) \dot{x}-\sin (\alpha+\beta) \dot{y}-(d+\ell \sin \beta) \dot{\theta}
$$$$
r \dot{\varphi}=\sin (\alpha+\beta) \dot{x}-\cos (\alpha+\beta) \dot{y}-(\ell \cos \beta) \dot{\theta}
$$

Thus, the matrix equation of the robot's inverse kinematics follows

$\left(\begin{array}{l}d_{1} \dot{\beta}_{1} \\ r_{1} \dot{\varphi}_{1} \\ d_{2} \dot{\beta}_{2} \\ r_{2} \dot{\varphi}_{2} \\ d_{3} \dot{\beta}_{3} \\ r_{3} \dot{\varphi}_{3}\end{array}\right)=\left(\begin{array}{ccc}-\cos \left(\alpha_{1}+\beta_{1}\right) & -\sin \left(\alpha_{1}+\beta_{1}\right) & -\left(d_{1}+\ell_{1} \sin \beta_{1}\right) \\ \sin \left(\alpha_{1}+\beta_{1}\right) & -\cos \left(\alpha_{1}+\beta_{1}\right) & -\left(\ell_{1} \cos \beta_{1}\right) \\ -\cos \left(\alpha_{2}+\beta_{2}\right) & -\sin \left(\alpha_{2}+\beta_{2}\right) & -\left(d_{2}+\ell_{2} \sin \beta_{2}\right) \\ \sin \left(\alpha_{2}+\beta_{2}\right) & -\cos \left(\alpha_{2}+\beta_{2}\right) & -\left(\ell_{2} \cos \beta_{2}\right) \\ -\cos \left(\alpha_{3}+\beta_{3}\right) & -\sin \left(\alpha_{3}+\beta_{3}\right) & -\left(d_{3}+\ell_{3} \sin \beta_{3}\right) \\ \sin \left(\alpha_{3}+\beta_{3}\right) & -\cos \left(\alpha_{3}+\beta_{3}\right) & -\left(\ell_{3} \cos \beta_{3}\right)\end{array}\right)\left(\begin{array}{c}\dot{x} \\ \dot{y} \\ \dot{\theta}\end{array}\right)$

Using the equation (2), we can calculate joint angle velocity and wheel velocity of each castor for a given mobile robot velocity. Moreover, for robot's dead reckoning, this equation is used. The above equation could be expressed like

$$
\dot{\mathbf{q}}=\mathbf{A}(\mathbf{q}) \dot{\mathbf{x}}
$$


To get the robot's velocity from encoder data of each castor, the following equation is used.

$$
\dot{\mathbf{x}}=\mathbf{A}^{\#}(\mathbf{q}) \dot{\mathbf{q}}
$$

where $\mathbf{A}^{\#}$ is pseudo-inverse of $\mathbf{A}$.

Then, $\mathbf{x}$ is the minimum norm solution among all solutions provided by the equation (3).

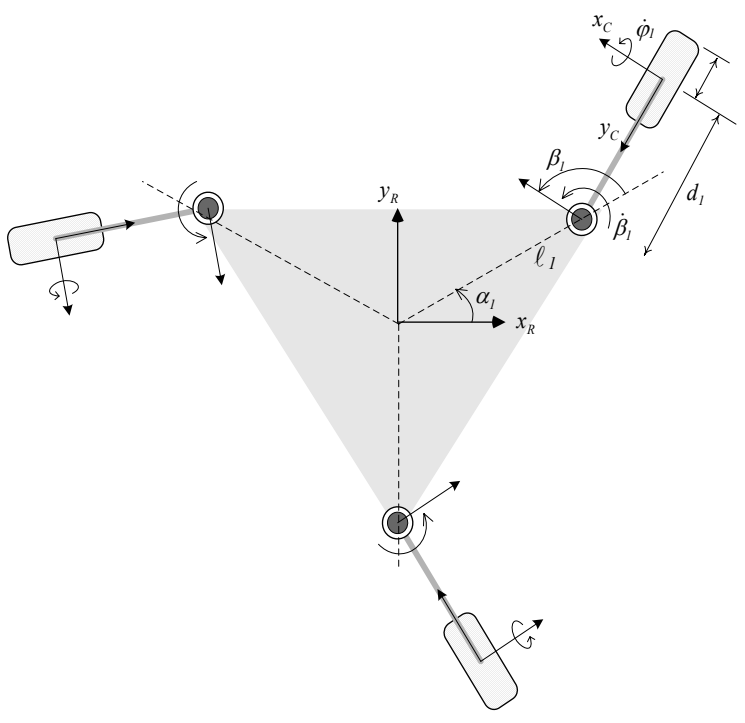

Figure 2. Configuration of three castor wheel mechanism.

\subsection{Localization}

Localization is to estimate a robot's coordinates in $\mathrm{x}$ $\mathrm{y}-\theta$ space, where $\mathrm{x}$ and $\mathrm{y}$ are the coordinates of the robot in a 2D Cartesian coordinate system, and $\theta$ is its orientation. Butler employed Markov localization, whose reliability and practical usefulness has been verified through the real applications for the tour guide robot in the museum[2]. Generally, the algorithm needs a lot of calculation time since it maintains probabilistic beliefs for whole possible states. Several remedies have been proposed to overcome this problem by the author who proposed the algorithm. Butler uses those remedies like selective update, various filters, and sensor lookup table models[2]. In addition, Butler employs an active area update technique. This technique is very useful in that unexpected areas in the map are removed at the beginning. Thus, it efficiently relieves the burden of calculation depending on the robot's task. For example, whole map area is divided into 5 small areas in the Figure 3. When the butler's mission is to guide a visitor into the front of door of a correspondent person, in this case, the robot doesn't have to go inside the rooms(The controller works in that way). Therefore, the active areas become Area 3 and Area 4. It means that the robot tries to find its location in those active areas. As you can guess from the size of the areas in the Figure 3, it can cut down, in this example, the calculation time of localization to about one third of the time that takes when it uses whole area.

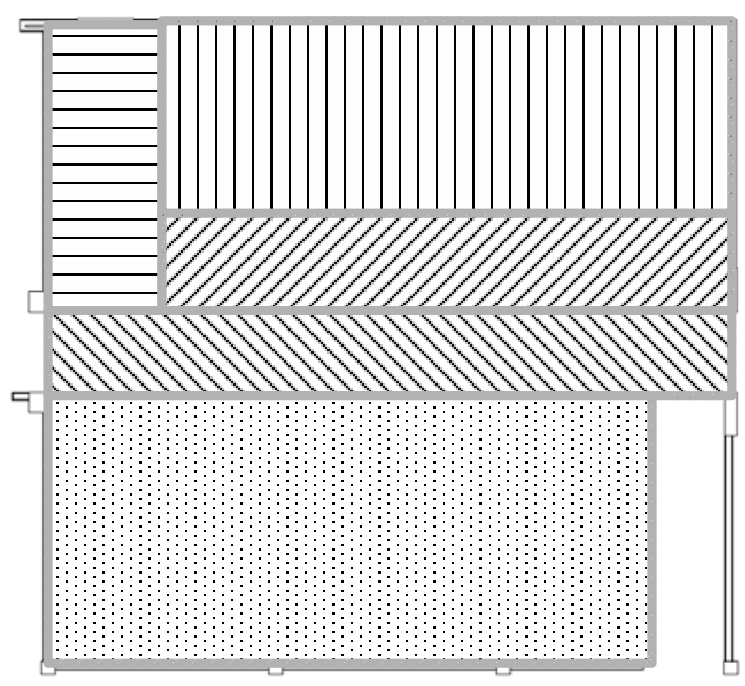

Figure 3. Small area example

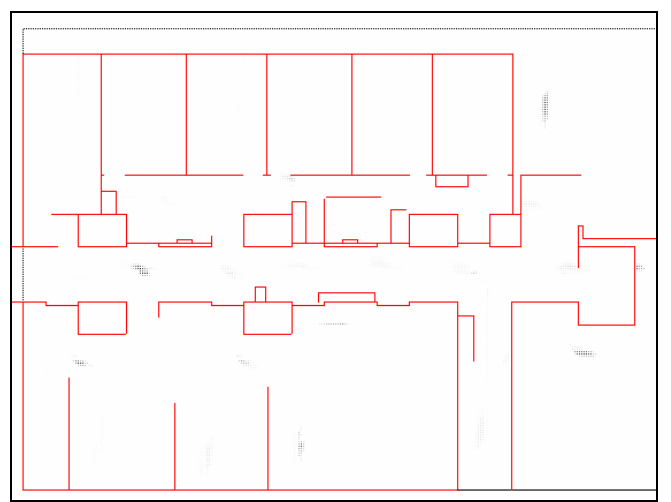

Figure 4. Markov localization from 9 laser scan data

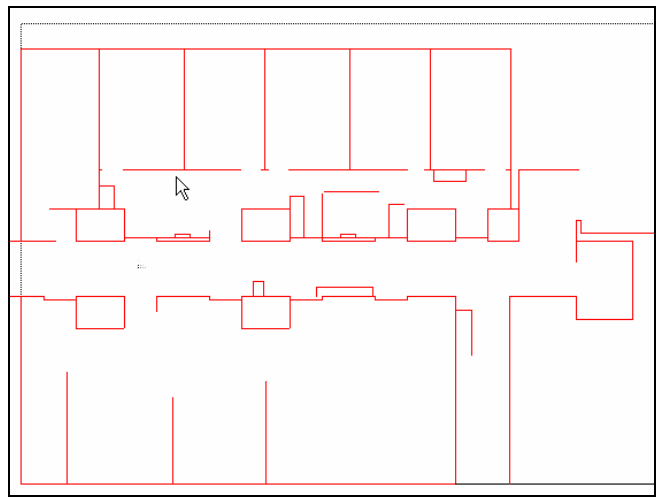

Figure 5. Markov localization from 18 laser scan data 


\subsection{Path-Planning}

Butler's path planner is based on dijkstra's algorithm, a well-known shortest path finding algorithm. The path planner generates paths from one node to another. The result paths are made in the form of sequential node numbers. For example, if the robot's near node is 8 and the destination node is 4 in figure 6 , then the result will become 8-9-2-3-4. Node information such as node's location and the distances between nearby nodes is saved in advance.

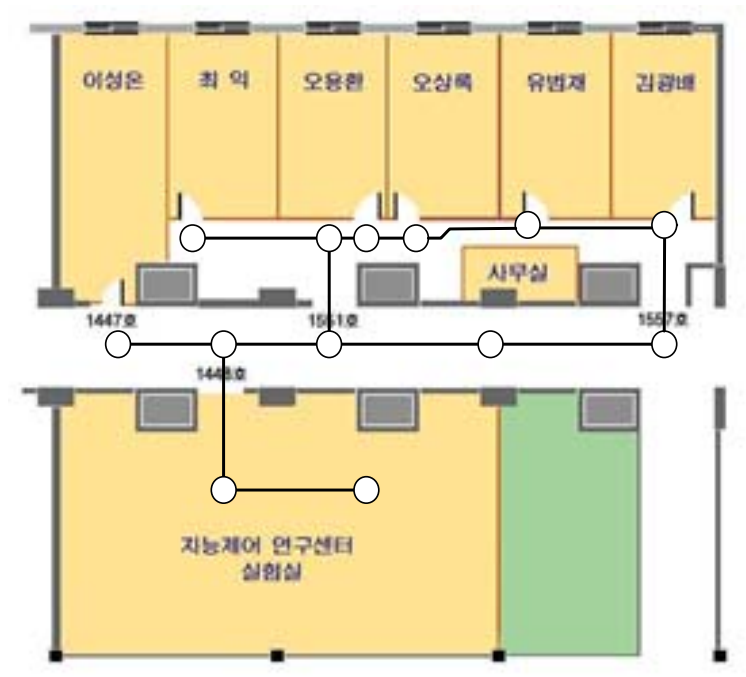

Figure 6. Node connections for path planning

\subsection{Local navigation (Obstacle avoidance)}

For local navigation between nodes, the Generalized Voronoi Graph(GVG)[1] is adopted. GVG is an effective tool for the guide robot because of its two major advantages. First, the GVG is a sensor based navigation which can adaptively react on the dynamic changes of the environment. For example, there could be a sudden human appearance and the robot should avoid the person reactively. This behavior can be implemented by using the incremental GVG construction algorithm which uses current sensor data for the next motion generation. Second, the GVG can be used for a semi-unstructured environment such as indoor corridor-like environment.

As a result, the GVG based navigation makes the robot move following the middle of empty space like in figure 7.

\section{HUMAN ROBOT INTERFACE}

Because Butler is designed to work as a service robot, its human-user interface (HRI) organization is an important part of our system. Working in the office environment, Butler usually interacts with the people, who are not robotic experts.

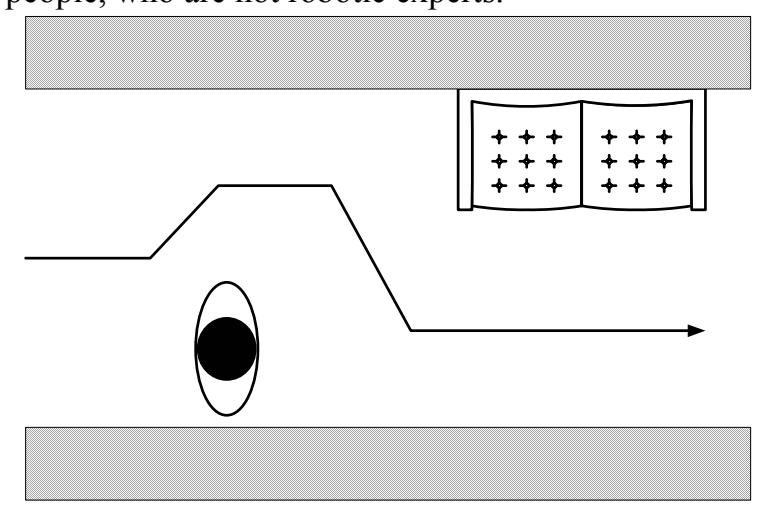

Figure 7. Expected trajectory of GVG

A good HRI system is the key for this robot to success. In this paper, we introduce and implement a multi-modal HRI system, that support all common HRI methods such as voice, vision, touch screen GUI. Moreover, we also design the Internet-based HRI, where users can interact with robot by using haptic devices with force feedback or by a professional web-based program. The system is designed so that all types of human-robot interaction can work simultaneously and in both directional. As users command Butler to do something, it can provide the information to the users via Voice, Vision, and the Internet. Besides directly interact with users, Butler also works as a mean to communicate users. After being asked about some one it knew, Butler searches the information and connects the user to the required person and allows communication (chat, voice, video conference) between them through its Internet HRI part.

Figure 9 shows the structure of the HRI system. We separate the interface part and the controlling part in two separate platforms and connect them via wireless network and serial communication. The internet-based HRI systems are existed in both platforms to support directly controlling of the hardware system or merge multi-modal interaction in the same input analyzing block.

Our experiments on the Butler system in the real office environment show that the interface is very convenient to the users. Butler show out to be good at understanding the requirements from the users and provides guiding services and informative, useful feedbacks via voice, video and GUI interface. Butler successfully guides people to the required places in most of the cases. Experiments with normal users also prove that the simultaneous multi-modal interaction system may improve the ability of exploring the system. 


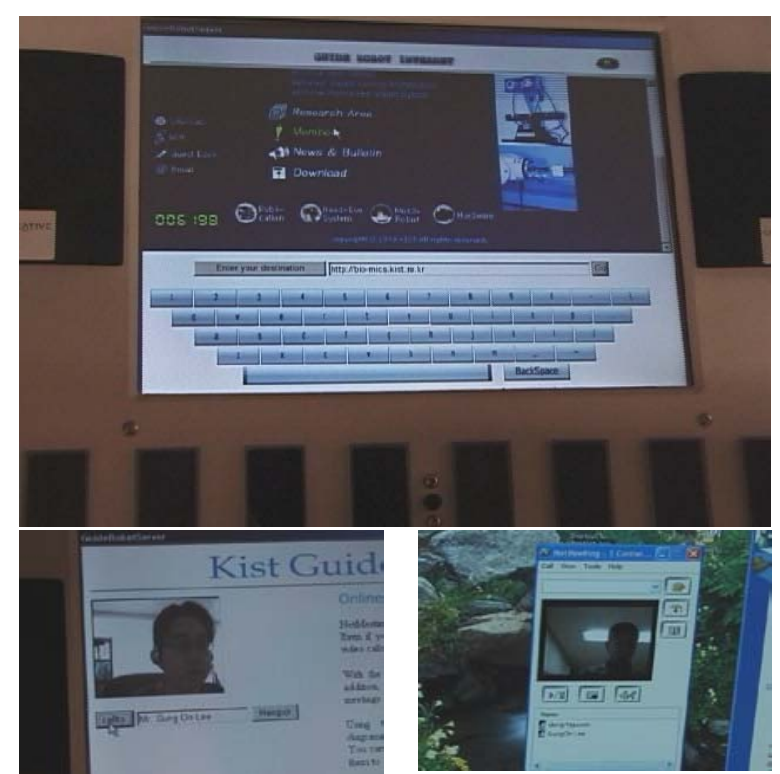

Figure 8. HRI (a) Internet service through touch screen $(b, c)$ Online chat service through voice and pc camera.

Using robot as a tool to communicate with people before guiding users via direct multi-media conferencing system seems stranger at first but turn out to be good in the long term experiments. We are also investigating another communication system to improve the interaction ability of our robotic system.

\section{CONCLUSION}

This paper described the hardware and the software of a visitor guide robot, which was successfully installed in the Korea Institute of Science and Technology. New ideas and solutions for challenges arising from the real guidance were addressed with overall explanation of each part. Through many real guidance demonstrations, we could have the following conclusions and future work includes those subjects.

1. The robot needs a fault-finding or a selfdiagnosing mechanism to make a fully autonomous robot in the case it loses its location completely or its batter level becomes low.

2. Voice service is much helpful than the service from the screen. Visitors usually do not like to stay near the robot when it moves. Thus, information through the screen was not easy to be given to the visitors when the robot is in the motion. Furthermore, people seemed to prefer talking to the robot more to touching it when they wanted to give it some order.
3. More friendly shape is preferable. This robot interacts with people who are generally not familiar with robotic systems. A friendly user-interface should be considered as important as the robot's functions when the robot needs to be used in public.

4. A good mapping method should be implemented. If the robot's working environment changes, we should reproduce a map for the robot. This could be a very time-consuming task when we do not have any CAD data for the new environment. Future research includes the integration of SLAM(simultaneous localization and mapping) which became already one of very popular research issues.

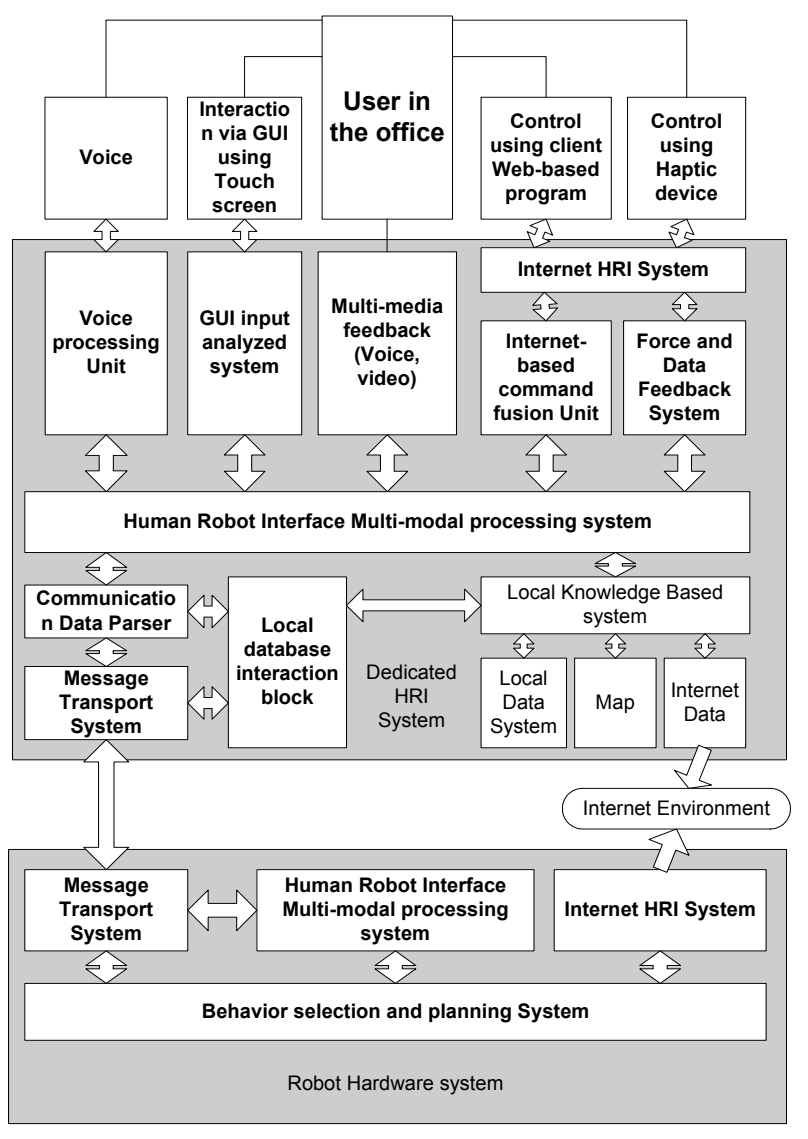

Figure 9. The structure of HRI system 


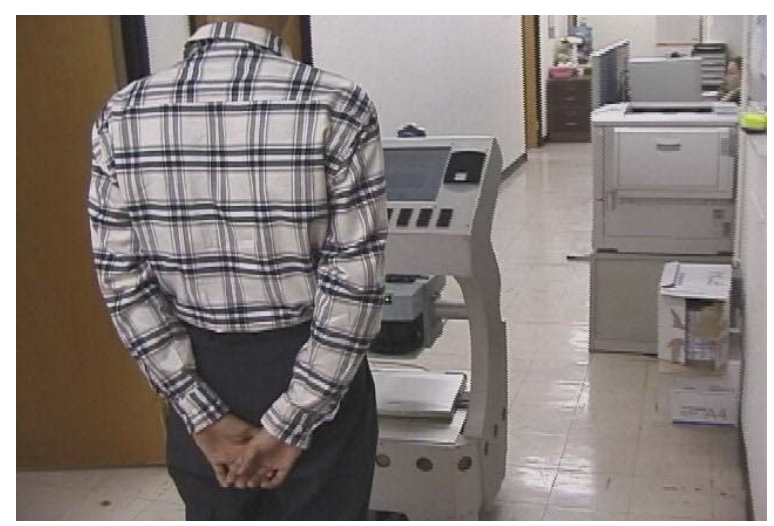

Figure 10. Guiding a visitor in the office environment

\section{REFERENCES}

[1] H.Choset and K.Nagatani, "Topological simultaneous localization and mapping(SLAM): Toward exact localization without explicit localization", IEEE Trans. Robotics and Automation, Vol. 17, No. 2, pp. 125-137, 2001.

[2] D. Fox, "Markov Localization: A Probabilistic Framework for Mobile Robot Localization and Navigation", PhD Thesis, University of Bonn, Germany, 1998.

[3] S. Thrun, M. Bennewitz, W. Burgard, A.B. Cremers, F. Dellarert, D. Fox, D. Hähnel, C. Rosenberg, N. Roy, J. Schulte, and D. Schulz, "MINERVA: A second generation mobile tour-guide robot", IEEE International Conference on Robotics and Automation(ICRA), pp. 1999-2005, 1999

[4] G. Campion, G. Bastin, and B. D’ Andrea-Novel, "Structural properties and classification of kinematics and dynamic models of wheeled mobile robots", IEEE Trans. On Robotics and Automation, Vol. 12, NO. 1, 1996.

[5] B. J. Yi and W. K. Kim "The Kinematics for Redundantly Actuated Omni-directional Mobile Robots", IEEE International Conference on Robotics and Automation(ICRA), pp. 2485-2492, 2000.

[6] B. J. You, M. Hwangbo, S. O. Lee, S. R. Oh, Y.D. Kwon, S. Lim, "Development of a Home Service Robot "ISSAC", IEEE International Conference on Intelligent Robots and Systems (IROS), pp. 26302635, 2003 